\title{
Complexion Time-Temperature-Transformation (TTT) Diagrams: Opportunities and Challenges
}

Onthida Schumachera, Christopher J. Marvela, Madeleine N. Kellyb, Patrick R. Cantwellc,, Richard P. Vincia, Jeffrey M. Rickmana, Gregory S. Rohrerb, Martin P. Harmera

a Department of Materials Science and Engineering, Lehigh University, 5 East Packer Avenue, Bethlehem PA 18015 USA

${ }^{b}$ Department of Materials Science and Engineering, Carnegie Mellon University, 5000 Forbes Avenue, Pittsburgh PA 15213 USA

c Department of Mechanical Engineering, Rose-Hulman Institute of Technology, 5500 Wabash Avenue, Terre Haute IN 47803 USA

*Corresponding author: Patrick R. Cantwell, (812) 877-8095, cantwep@rose-hulman.edu

\begin{abstract}
Grain boundaries and other interfaces can undergo complexion transitions from one thermodynamic state to another, resulting in discontinuous changes in interface properties such as diffusivity, mobility, and cohesive strength. The kinetics of such complexion transitions has been largely overlooked until recently. Just as with bulk phase transformations, complexion transition kinetics can be represented on time-temperaturetransformation (TTT) diagrams. An experimental complexion TTT diagram is presented here for polycrystalline Eu-doped spinel annealed at 1400 to $1800{ }^{\circ} \mathrm{C}$. This material developed a microstructure with a bimodal grain size distribution, indicating that a complexion transition occurs within this temperature range. The time and temperature dependence of this complexion transition was analyzed and used to produce a grain-boundary complexion TTT diagram for this system. Complexion TTT diagrams have the potential to be remarkably useful tools for manipulating the properties of internal interfaces in polycrystalline metals and ceramics. The development of experimental complexion TTT diagrams is likely to have an important impact on the field of grain-boundary engineering, and hence the development of these experimental diagrams should be an intense area of focus in the coming years.
\end{abstract}

\section{KEYWORDS}

TTT diagram

Grain Boundary Complexions

Magnesium Aluminate Spinel

Abnormal Grain Growth 


\section{Complexion Time-Temperature-Transformation Diagrams}
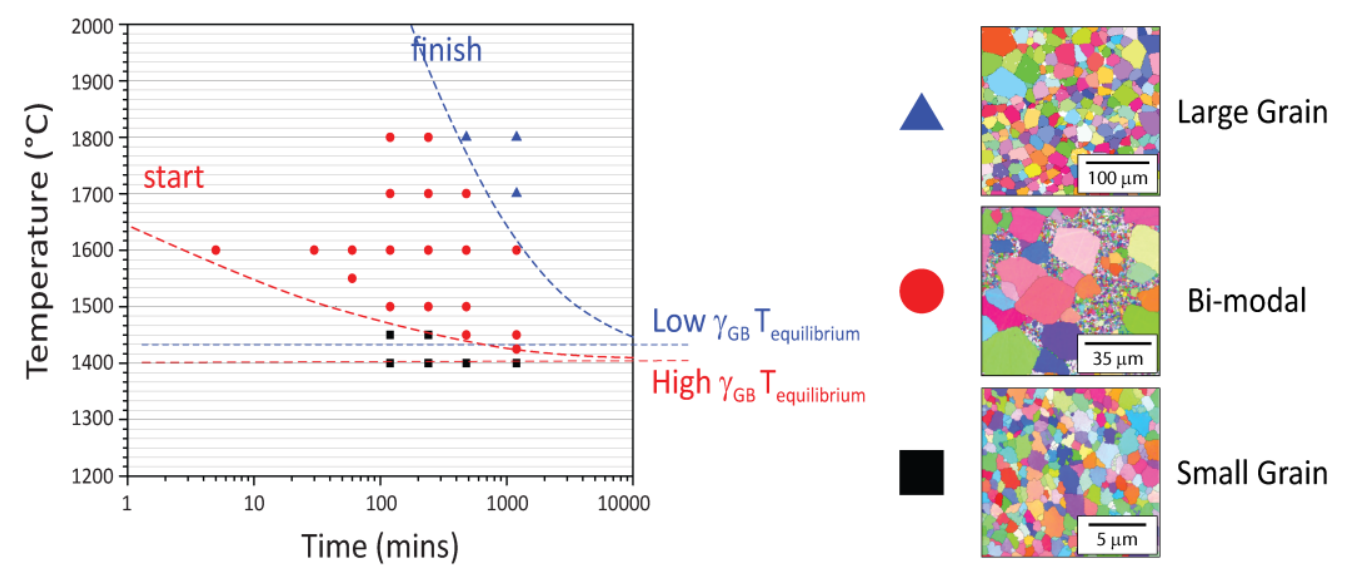

\section{Introduction}

Davenport and Bain made a pivotal breakthrough in our understanding of bulk phase kinetics when they introduced the first experimental time-temperature-transformation (TTT) diagrams in 1930, which were based on a detailed and extensive study of steel phase transformation kinetics [1]. These TTT diagrams were revolutionary because they offered a simple and easily understood visual representation of a complicated and often mysterious process. The power and utility of TTT diagrams was immediately recognized, and the subsequent widespread usage of these diagrams enabled rapid progress in the processing and heat treatment of steel and other alloys.

Interfaces such as grain boundaries can also exhibit phase-like behavior [2], transforming from one equilibrium state to another as a function of thermodynamic variables in a process known as a complexion transition [3]. Complexion transitions are important because they are often accompanied by discontinuous changes in grain-boundary properties such as mobility, diffusivity, and cohesive strength, and hence these transitions can dramatically 
influence the macroscopic properties of materials [3]. Although the study of complexion transitions dates back decades, as summarized in recent review articles [3,4], the kinetics of complexion transitions has been largely overlooked by the materials community until very recently. Just as with bulk phase transformations, complexion transitions take time to occur, and therefore their kinetics can be represented on TTT-style diagrams. The first experimental complexion TTT diagrams were recently reported based on grain growth studies of polycrystalline $\mathrm{Y}_{2} \mathrm{O}_{3}$ and $\mathrm{Al}_{2} \mathrm{O}_{3}$ [5]. These diagrams clearly show the combinations of time and temperature at which grain-boundary complexion transitions occur, unifying and displaying a large data set in a readily understood format.

Although grain-boundary complexion TTT diagrams are analogous to bulk phase TTT diagrams in many ways, there are important differences between them. For example, bulk phase TTT diagrams typically only show the transformation curve for the cooling transformation which, in its simplest form, is described by a C-shaped transformation band (see Fig. 1(a)) that results from a competition between nucleation and growth rates. This competition produces a minimum transformation time at a particular sub-cooling temperature, often referred to as the nose of the TTT curve. On the other hand, during the heating transition, the nucleation and growth rates both increase as temperature increases and hence augment each other, leading to a transformation that occurs ever more rapidly with increasing temperature. A heating transition diagram therefore lacks the classic nose feature of the cooling diagrams. An isothermal heating transformation is shown schematically in Figure 1(b). Although cooling TTT diagrams are typically most useful for bulk phases due to their importance in heat treatment operations, heating TTT diagrams are arguably more important for the study of grain-boundary complexion transitions. If the heat treatment temperature exceeds the complexion transition temperature, discontinuous jumps in the grain-boundary diffusivity and mobility can lead to runaway grain growth (e.g., during sintering), enhanced oxidation rates, and other potentially undesirable effects. Thus, by understanding the temperature and time limits within which grain-boundary complexion transitions will not occur, enhanced protocols for materials processing under various service conditions can be developed. 
(a)

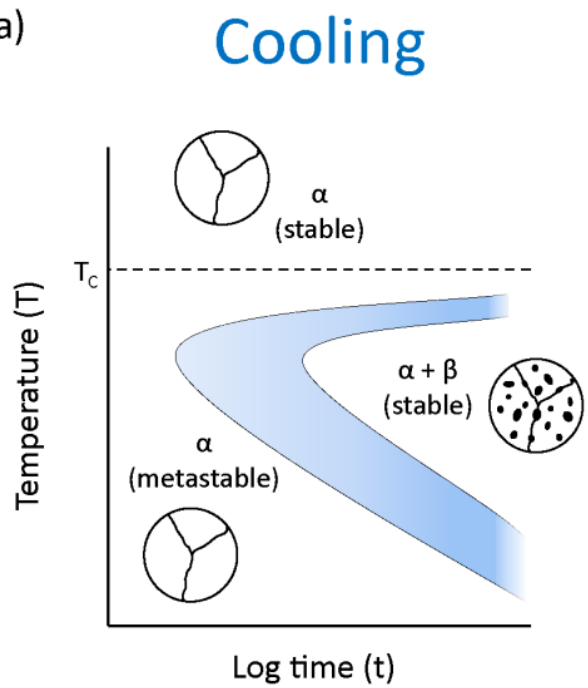

(b)

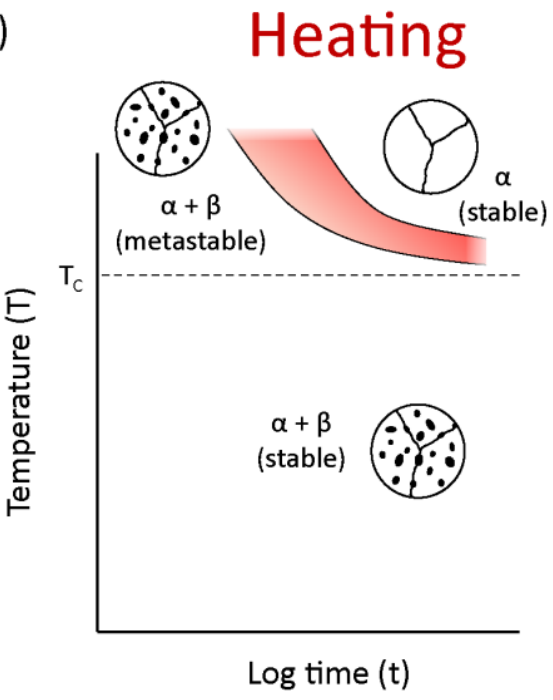

Figure 1: Schematic bulk phase TTT diagrams for a hypothetical system that transitions between a single $\alpha$ phase and $\alpha+\beta$ phases as a function of temperature: (a) Isothermal cooling and (b) isothermal heating.

It is apparent from Figure 1 that the regions of bulk phase metastability are reversed for cooling and heating TTT diagrams. In the TTT cooling diagram in Figure 1(a), the $\alpha$ phase is metastable below $\mathrm{T}_{\mathrm{C}}$ for short times, whereas in the TTT heating diagram in Figure 1(b), the $\alpha+\beta$ microstructure is metastable above $\mathrm{T}_{\mathrm{C}}$ for short times. Complexion TTT diagrams exhibit analogous regions of metastability [5], suggesting that they undergo nucleation and growth processes similar to those characteristic of bulk phases. One signature of a grain-boundary complexion transition is the appearance of abnormally large grains [3,6], and experiments have shown that the number density of abnormal grains increases exponentially with temperature [7], an observation that is consistent with a nucleation and growth mechanism. Although no direct evidence exists yet that grain-boundary complexion transitions occur via a nucleation and growth process, it has been shown experimentally that surface complexion transitions do involve nucleation and growth processes [8]. It therefore seems reasonable that an analogous nucleation and growth process occurs during grain-boundary complexion transitions, although more research is needed in this area to elucidate the true behavior. 
Another fundamental difference between bulk phase TTT diagrams and grain-boundary complexion TTT diagrams is that grain boundaries have five additional degrees of thermodynamic freedom as compared to bulk phases, i.e. the five macroscopic parameters that describe grain misorientation and grain-boundary inclination (three parameters for grain misorientation, and two for grain-boundary plane inclination). Complexion transition kinetics can vary from one grain boundary to another because grain boundaries of different character exist in different thermodynamic states owing to difference in interfacial atomic geometry. It has been shown that complexion transitions preferentially occur on higher energy interfaces [9] at shorter annealing times than transitions on lower energy interfaces [5]. Therefore, the grain-boundary character distribution (GBCD) and the resultant grainboundary energy anisotropy present in a polycrystalline material may lead to grainboundary complexion transitions occurring at a variety of different times and temperatures. For example, within a given polycrystalline specimen, some high energy grain boundaries might undergo a complexion transition at relatively low temperatures and short times, while other grain boundaries with lower energies might not transition until higher temperatures and longer times. Furthermore the lowest energy grain boundaries might not undergo a complexion transition prior to melting. This variety of kinetic behavior caused by the structural and energetic anisotropy of grain boundaries means that a complexion TTT diagram should, in principle, contain a different transformation band for each type of grain boundary. This situation is shown schematically for two grain boundaries of different character in the isothermal heating complexion TTT diagram in Figure 2. 


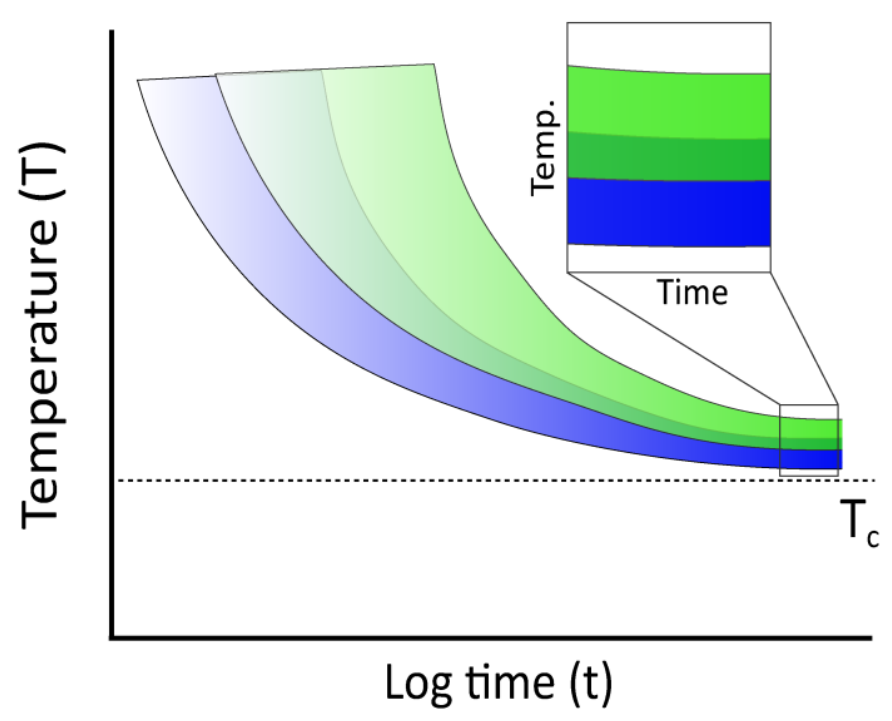

Figure 2: Schematic isothermal heating complexion TTT diagram for two types of grain boundaries with different grain-boundary character. The transition of the high-energy grain boundary, depicted by the blue transition band, occurs at shorter times and lower temperatures, while the slower transition of the low-energy grain boundary is depicted by the green band. In the example depicted, there is a region of overlap between the two bands but this need not be the case.

Of course, a complexion TTT diagram that contains a transformation curve for each different type of grain boundary in a polycrystalline material would be incredibly complex and would therefore have limited utility. In practical cases, a subset of grain boundaries will undergo a complexion transition under similar conditions [3]. Therefore, it will often be preferable to group together subsets of grain boundaries with similar behavior when plotting grainboundary complexion TTT diagrams, such that each subset has its own transformation curve.

Grain-boundary complexion TTT diagrams are advantageous for depicting the kinetics of complexion transitions because they illustrate, in a readily understood format, the complex relationship between annealing time, temperature, and properties that depend on these transitions. Hence, one can imagine that complexion TTT diagrams will be useful, for example, for controlling grain size during annealing and sintering, and will have additional applications in other situations in which complexion transitions play a key role. The ability 
to understand and control the kinetics of complexion transitions will be critical to advancing the state of the art in polycrystalline metals and ceramics, since their behavior is so often dominated by interface properties. We therefore believe that the development of complexion TTT diagrams is an important area for current and future research as it will have a positive impact on the field of grain boundary engineering $[10,11]$ in particular, and on advancing the state of the art in the processing and performance of polycrystalline materials in general.

\section{PROOF OF CONCEPT}

As a proof of concept, a complexion TTT diagram has been constructed based on experimental data from a Eu-doped spinel system. This system was chosen for this purpose because it exhibits a strong propensity for abnormal grain growth that is attributed to a grain-boundary complexion transition associated with Eu boundary segregation, and because it was observed to possess significant anisotropy in grain-boundary energy and grain-boundary character. In this system, the onset of abnormal grain growth and changes in grain boundary anisotropy were identified for a range of annealing temperatures. The appearance of an abnormally large grain in the microstructure was taken as a signature of a complexion transition, indicating that the grain boundary surrounding the abnormally large grain had undergone a change in structure and chemistry that resulted in a dramatic increase in grain-boundary mobility. This interpretation of abnormal grain growth has been supported by many previous experimental studies, e.g. [3,6,7]. As shown in Figure 3(a), a sample annealed at $1400^{\circ} \mathrm{C}$ exhibits normal grain growth with a nearly isotropic grain-boundary plane distribution, whereas a sample annealed at $1600{ }^{\circ} \mathrm{C}$ exhibits abnormal grain growth that has a significant preference for $\{111\}$ grain-boundary planes. Changes in the grain-boundary plane distribution have previously been linked to complexion transitions $[12,13]$. 
(a)
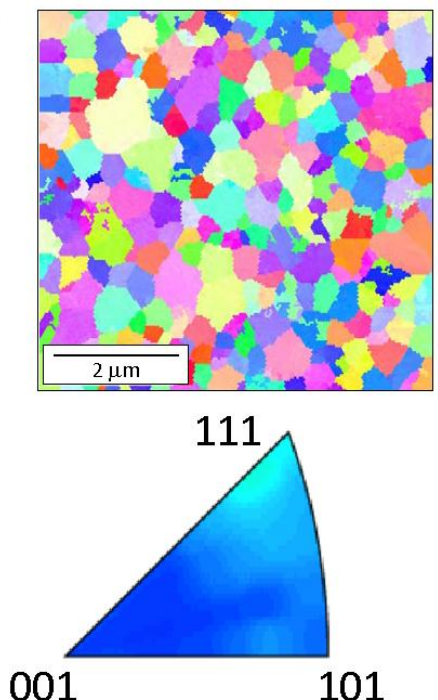

(b)

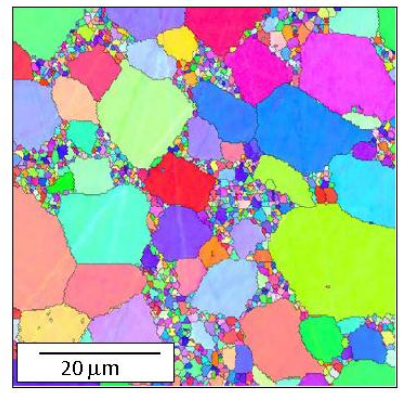

111

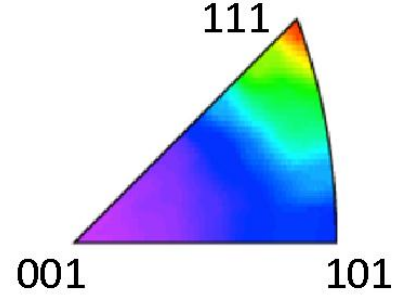

$\begin{array}{llllllllllll}0.7 & 0.8 & 0.9 & 1.0 & 1.1 & 1.2 & 1.3 & 1.4 & 1.5 & 1.6 & 1.7\end{array}$

Multiples of Random

Figure 3: EBSD micrographs (top) and the distributions of grain-boundary planes in the crystal reference frame (bottom) of polycrystalline Eu-doped spinel annealed at temperatures of (a) 1400 ${ }^{\circ} \mathrm{C}$ and (b) $1600{ }^{\circ} \mathrm{C}$. The specimen annealed at $1400{ }^{\circ} \mathrm{C}$ has a unimodal grain size distribution while the specimen annealed at $1600{ }^{\circ} \mathrm{C}$ has a bimodal grain size distribution, indicating that grain-boundary complexion transitions have occurred.

\subsection{Experimental Procedure}

Dense polycrystalline Eu-doped (500 wt-ppm) spinel samples were fabricated by sparkplasma sintering at $1200{ }^{\circ} \mathrm{C}$ for one hour with $40 \mathrm{MPa}$ applied pressure. A cylindrical sample of $20 \mathrm{~mm}$ diameter with near theoretical density was obtained, and smaller pieces $\left(\sim 5 \mathrm{~mm}^{3}\right)$ were sectioned and cleaned thoroughly to remove any contamination. The smaller pieces were annealed at various temperatures between $1400{ }^{\circ} \mathrm{C}$ and $1800{ }^{\circ} \mathrm{C}$ for different periods of time between 0 and 20 hours in a $5 \%$ hydrogen balanced nitrogen reducing atmosphere. In this study, a 0 hour annealing time refers to the scenario in which the samples were heated to the annealing temperature and subsequently cooled without dwelling at the annealing temperature. Following annealing, all samples were polished utilizing various diamond suspensions and cleaned thoroughly to remove residual materials from polishing. An electron backscattered diffraction (EBSD) technique was 
utilized to monitor the microstructure evolution of the doped spinel as a function of time and temperature. An EDAX Hikari EBSD camera that was attached to a Hitachi 4300N scanning electron microscope (SEM) was utilized for this purpose. The relative areas of different grainboundary planes in the crystal reference frame (see Fig. 3) were calculated stereographically [14]. The change in the distribution of grain-boundary planes, together with the presence of abnormal grains, are indicators of a complexion transition.

\subsection{Results and Discussion}

A typical bimodal EBSD map and grain-size histogram are presented in Figure 4. At least 1000 grains were measured from several EBSD maps to produce the histogram. As seen in the histogram, the maximum occurs for grains that were less than $3 \mu \mathrm{m}$ in diameter. More than half of the grains fall into this category. At the relatively low magnification of Figure 4(a), the small granular regions are noisy because of the choice of step size utilized for collecting the EBSD data. The bimodality of this microstructure, as with every other bimodal microstructure considered here, is hypothesized to be an indication that a complexion transition has occurred. The premise is that at an earlier stage of annealing (i.e. a time prior to the recorded EBSD map), complexion transitions occurred along some grain boundaries and thereby enhanced the mobility of those boundaries. Thus, the grains encompassed by the transitioned complexions grew at a faster rate than the rest of the matrix. Given this hypothesis, and assuming complexion transitions are first-order in nature, increasing the annealing time or temperature will increase the number of abnormally large grains at the cost of the smaller grain population. 

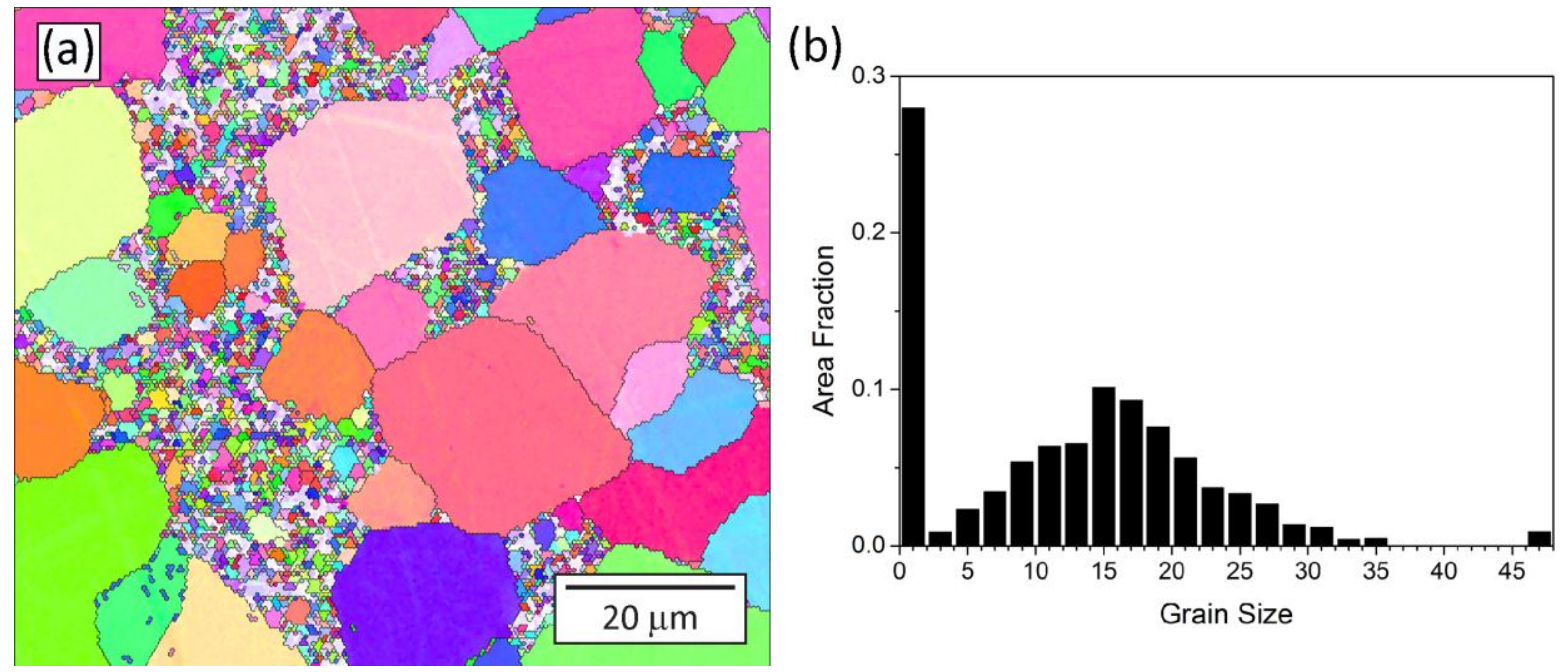

Figure 4: (a) A representative EBSD map, and (b) grain size histogram of bimodal Eu-doped spinel.

To investigate the temperature dependence of the nucleation rate, several Eu-doped spinel samples were annealed at various temperatures and times. For each resulting microstructure, the grains were partitioned into "small" and "large" groups with diameters less than or greater than 3 $\mu \mathrm{m}$. This cut-off value was chosen based on the estimated maximum size of the small grain population observed in the majority of microstructures. The partitioned EBSD data are shown in Figure 5, with abnormally large grains displayed in color and small (i.e., normal) grains in black. From the partitioned EBSD maps, the relative fractions of the small and large grain populations were calculated. In the samples annealed for $0 \mathrm{hrs,}$ no abnormally large grains were observed in the $1500{ }^{\circ} \mathrm{C}$ and $1600{ }^{\circ} \mathrm{C}$ samples, whereas abnormal grains covered $4 \%$ of the image area in the $1700{ }^{\circ} \mathrm{C}$ sample. As the dwell time was prolonged at $1500{ }^{\circ} \mathrm{C}$, the area fraction of abnormal grains increased progressively, and impingement between abnormal grains was not significant. In contrast, at $1600{ }^{\circ} \mathrm{C}$ and $1700{ }^{\circ} \mathrm{C}$, impingement of abnormal grains was observed after 2 hours of annealing. The area fractions for larger grain population were $44 \%$ and $64 \%$, respectively, at $1600{ }^{\circ} \mathrm{C}$ and $1700{ }^{\circ} \mathrm{C}$. Furthermore, and as expected, the area fractions increased with annealing time at $1600{ }^{\circ} \mathrm{C}$ and $1700{ }^{\circ} \mathrm{C}$. 


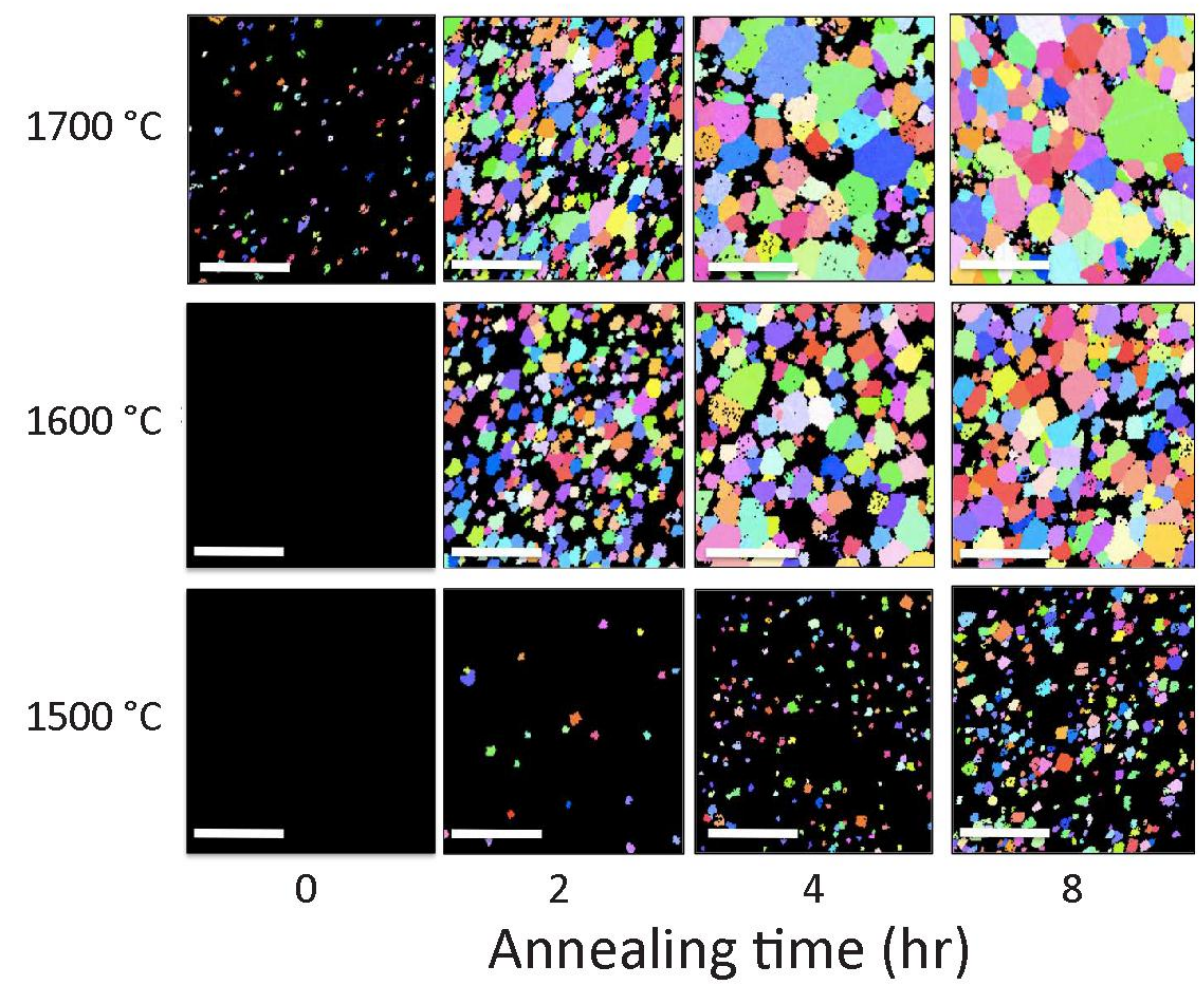

Figure 5: A time-temperature matrix of partitioned EBSD maps. Small grain regions are shown in black and the scale bars represent $70 \mu \mathrm{m}$.

While the area fraction of abnormally large grains is not a perfect measure of the fraction of grain boundaries that have undergone a complexion transition, the two quantities are strongly correlated. Therefore, the evolving area fraction of abnormal grains is a useful approximation for highlighting the kinetics of complexion transitions, and the area fraction of the abnormally large grains was extracted from samples treated at three different annealing temperatures $\left(1500^{\circ} \mathrm{C}\right.$, $1600^{\circ} \mathrm{C}$ and $1700^{\circ} \mathrm{C}$ ) for various periods of time for this purpose. The kinetics of nucleation and growth in bulk systems can be quantified using the Johnson-Mehl-Avrami equation [15], and we will also employ this description here. In this approach, the transformed area fraction, $A_{t}$, for grains after a time $t$ is given by

$$
1-A_{t}=\exp \left(-k t^{n}\right)
$$


where $k$ is a reaction constant and $n$ is the Avrami exponent. Equation (1) may be rewritten in a form that is convenient for analysis and is given by

$$
\ln \left[\ln \frac{1}{1-A_{t}}\right]=\ln k+n \ln t
$$

Figure 6 shows the fitted Avrami curves of the $1500{ }^{\circ} \mathrm{C}, 1600{ }^{\circ} \mathrm{C}$ and $1700{ }^{\circ} \mathrm{C}$ sample sets. The Avrami parameters $n$ and $k$ were determined by fitting Equation 2 to the data; the results are summarized in Table 1. We note that the Avrami analysis as employed here pertains strictly to bulk systems. Nevertheless, its application to complexion transitions may reveal some relevant characteristics of the nucleation and growth process. In particular, there are several possible scenarios that would lead to $\mathrm{n} \approx 1$, including nucleation site saturation and nucleation/growth in a reduced spatial dimensionality. However, given the limited data available as to the temporal dependence of the number of boundaries transformed (see Figure 6), a more definitive interpretation must await additional time-transformation data and, in some circumstances, may require a more general analysis of nucleation and growth kinetics [16,17].

Table 1: Avrami parameters $k$ and $n$ determined by fitting Equation 2 to the experimental data.

\begin{tabular}{|c|c|c|}
\hline & $k$ & $n$ \\
\hline $1500{ }^{\circ} \mathrm{C}$ & $0.00005 \mathrm{~min}^{-1.39}$ & 1.39 \\
\hline $1600{ }^{\circ} \mathrm{C}$ & $0.00180 \mathrm{~min}^{-1.10}$ & 1.10 \\
\hline $1700{ }^{\circ} \mathrm{C}$ & $0.00517 \mathrm{~min}^{-1.03}$ & 1.03 \\
\hline
\end{tabular}

The fitted Avrami curves were then extrapolated to establish the complexion transition start (1\%) and finish (99\%) times for each temperature. 


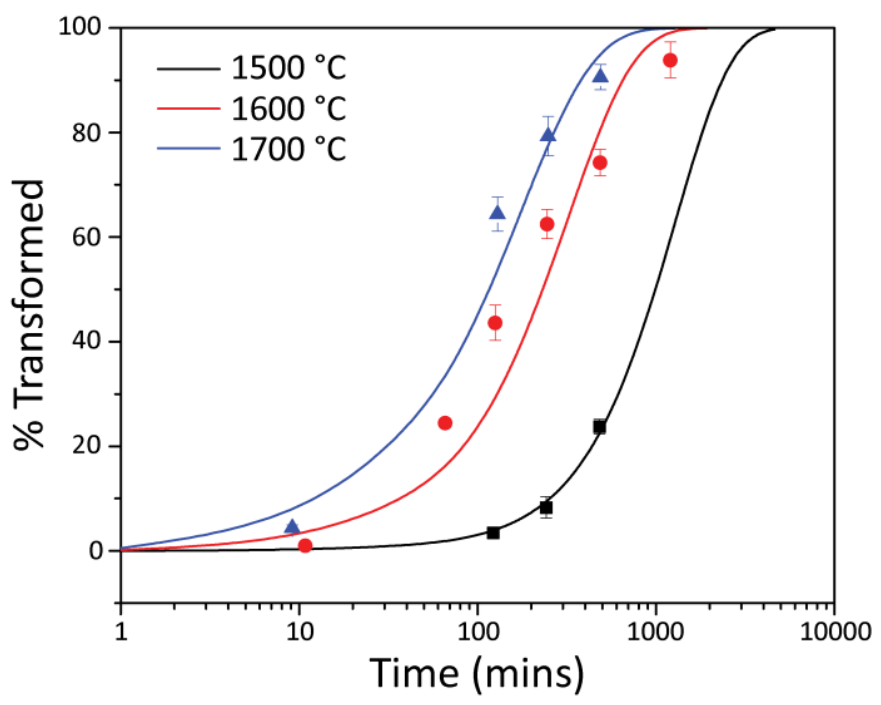

Figure 6: Avrami plots representing the percentage of grain boundaries that have undergone a complexion transition as a function of time at temperatures of 1500,1600 , and $1700{ }^{\circ} \mathrm{C}$. Avrami curves are overlaid on the experimental data points to better illustrate the transition kinetics.

An alternative and perhaps more practical representation of complexion kinetics is a complexion TTT diagram. In this representation, the temperature $(T)$ is plotted as a function of time $(t)$ at fixed transformation fraction. The complexion TTT diagram for $500 \mathrm{wt}$-ppm europiumdoped spinel is presented in Figure 7. As is evident from the figure, the diagram has three distinct time-temperature regimes: squares.

Regime I: Grains are $<3 \mu \mathrm{m}$ in diameter. This microstructure is represented by black

Regime II: Grain size is bimodal. This microstructure is represented by red circles.

Regime III: Grains are > $3 \mu \mathrm{m}$ in diameter. This microstructure is represented by blue triangles.

Regimes I and III are characterized by a unimodal grain size microstructure, with the main observable microstructure difference being the average grain size. At a given temperature in the complexion TTT diagram in Figure 7, the dashed red line (1\% line) between regime I and regime II represents the time after which grain-boundary complexion transitions have just begun to occur, while the dashed blue line (99 \%) line separating regimes II and III represents the time at which nearly all grain boundaries have transitioned (or the non-transitioned boundaries have been eliminated by the growth of grains surrounded by transformed boundaries). To illustrate the combinations of time and temperature that separate these three regimes, curves were drawn 
through points in time calculated based on the curves in Figure 6, i.e. the times at which $1 \%$ and $99 \%$ of the boundaries have transformed at temperatures of 1500,1600 , and $1700{ }^{\circ} \mathrm{C}$.

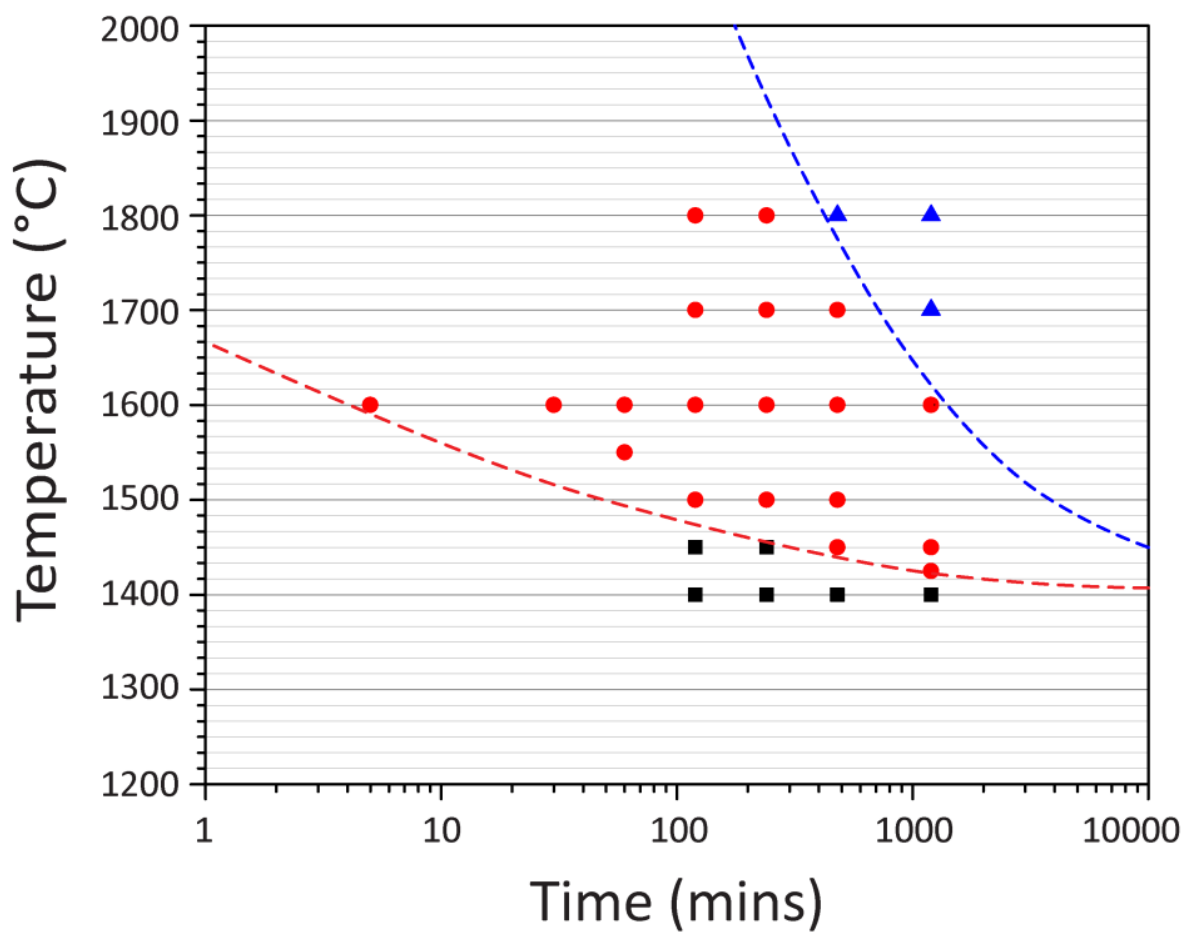

Figure 7: Grain-boundary complexion time-temperature-transformation (TTT) diagram of Eudoped spinel derived from experimental data.

As pointed out in the following discussion of future prospects, because the complexion TTT diagram in Figure 7 was produced by using AGG as a proxy for complexion transitions, it cannot fully isolate the kinetics of complexion transitions from the kinetics of abnormal grain growth. The net effect is that the complexion transition times depicted in Figure 7 are somewhat longer than the actual time required for the complexion transitions themselves to occur. This time disparity is caused by the additional time it takes for grains affected by the complexion transitions to grow large enough to be recognized as being abnormally large. Developing experimental techniques that more accurately measure the time required for complexions to nucleate and grow is a critical challenge that must be addressed to advance the field. 


\section{FUTURE PROSPECTS}

\subsection{Opportunities}

Complexion TTT diagrams summarize the kinetics of complexion transitions and can therefore be used to design optimized heat treatments. These complexion TTT diagrams can be used to engineer new classes of materials using a complexion-informed approach to accomplish any number of use-inspired objectives. In materials systems in which grain-boundary complexion transitions result in abnormal grain growth, as in the Eu-doped spinel proof-of-concept system above, it is theoretically possible to create single crystals, bimodal microstructures, and coarsening-limited (e.g., nanocrystalline) microstructures by selecting an appropriate heat treatment strategy. Most importantly, all of these microstructures can be produced from the same starting microstructure. Figure 8 schematically illustrates the heat treatments required to produce each of the aforementioned final microstructures, and Figure 9 shows three example microstructures from the previously discussed spinel experiments. For clarity, only the start and $1 \%$ transformation lines are shown to highlight the significance of the starting transformation curve. Each optimization process is also described briefly below. 


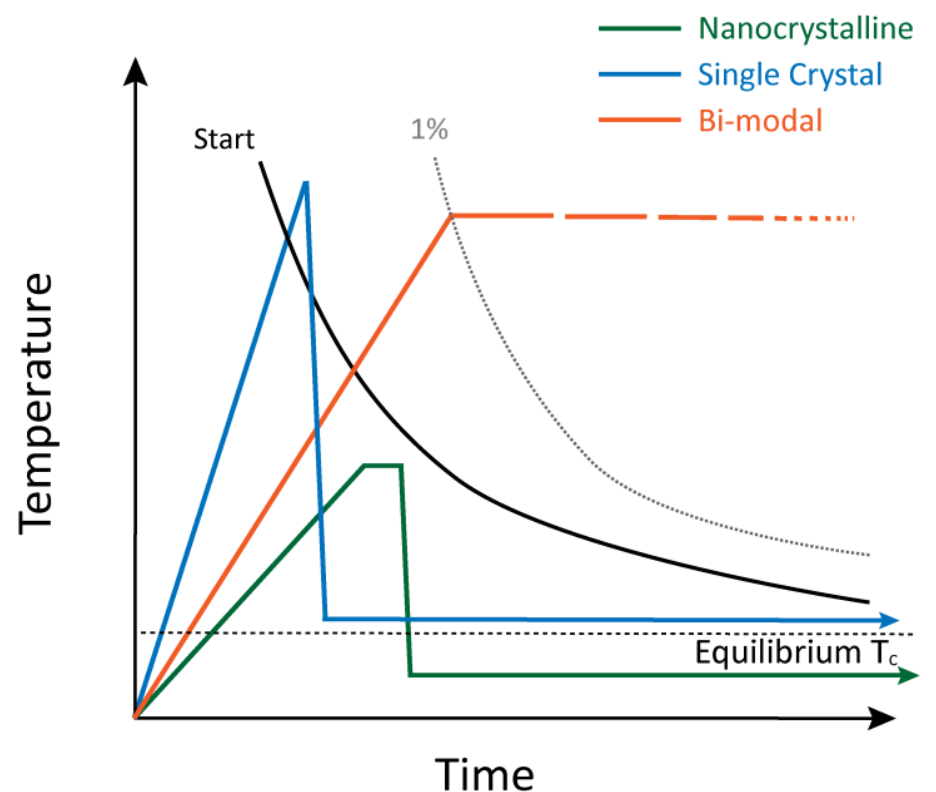

Figure 8: Schematic illustration of theoretical heat treatments to produce single crystals, bimodal microstructures, and coarsening-limited (e.g., nanocrystalline) materials.

1. To achieve coarsening-limited materials, first dwell at a temperature just below the transformation start line to maximize early stage densification and avoid transitions to higher mobility grain-boundary complexions. Next, drop the temperature to below the equilibrium complexion transition temperature, $\mathrm{T}_{\mathrm{C}}$, to complete the densification process while avoiding any undesired complexion transitions that would cause accelerated grain growth. Figure 9a shows a unimodal microstructure processed in this manner consisting of sub-micron grains.

2. To achieve a bimodal microstructure, transform a desired portion of the grain boundaries at an elevated temperature and then drop below the equilibrium temperature to inhibit additional nucleation events. Figure $9 \mathrm{~b}$ shows a bi-modal microstructure processed in this manner containing "small" and "large" grain populations with average grain sizes of $0.5 \mu \mathrm{m}$ and $10 \mu \mathrm{m}$, respectively.

3. To achieve a single crystal, first apply a heat treatment that narrowly crosses the transformation line in order to nucleate a single large grain. Next, immediately cool to just above the equilibrium transformation temperature and then anneal to allow a large 
monopolizing grain to consume the smaller matrix grains of the microstructure. Figure 9c illustrates a large-grain island cluster indicating the potential for single crystal seed nucleation.
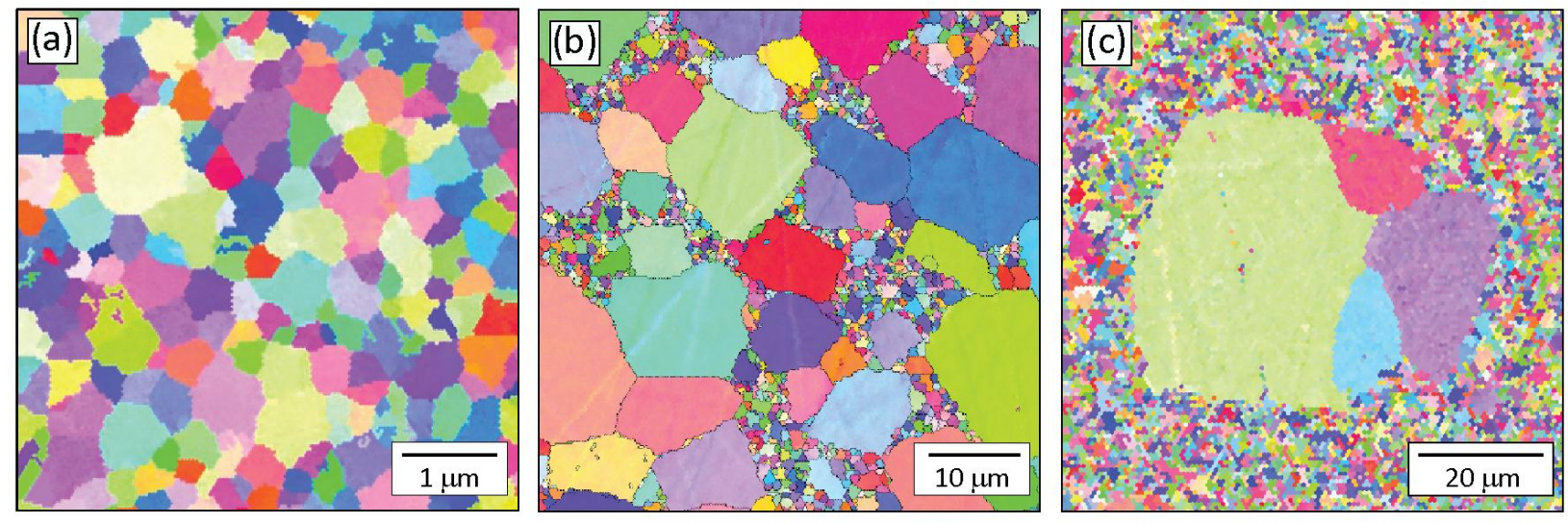

Figure 9: Three proof-of-concept microstructures illustrating (a) sub-micron grains, (b) a bimodal microstructure, and (c) a near single crystal growing in the microstructure.

The final microstructure strongly depends on whether the transformation line is crossed and, if so, the length of time that the sample is held in the transformation region. For instance, merely crossing the transformation line before holding at a temperature lower than $\mathrm{T}_{\mathrm{C}}$ could flip the microstructure from nanocrystalline to a single crystal. The ability to engineer three significantly different microstructures from the same starting material is also technologically very advantageous. Each microstructure class provides useful properties: single crystal sapphire can be used for high strength armor windows [18] or substrates for LED applications [19], a bimodal microstructure in many materials can enhance toughness as compared to a microstructure with a unimodal grain size distribution [20], and ultra-fine grain alumina can have higher strength and ductility as compared to coarse-grained alumina [21].

These novel complexion-informed heat treatments also demonstrate new mechanisminformed approaches to producing bulk sub-micron grain materials and large-scale single crystals. For example, Chen et al. proposed a two-step sintering method to fabricate nanocrystalline ceramics that is similar to the proposed heat treatment above (i.e., the green line in Figure 8), but did not attribute the associated mechanism to the careful avoidance of a complexion transition at 
the higher annealing temperature; rather, triple-junction drag was proposed as the dominant mechanism at low temperatures [22]. With regard to single-crystal conversion, other solid-state conversion techniques require either a seed crystal [23] or extreme processing conditions to grow large single crystals akin to the Czochralski process [24]. By contrast, our proposed conversion mechanism involves the nucleation of a "seed" crystal in-situ and then exploits controlled abnormal grain growth to eradicate the smaller grains.

\subsection{Challenges and Recommendations}

The field of complexion kinetics is still in its infancy and many challenges must be overcome to construct, understand, and fully utilize complexion TTT diagrams. First and foremost, the protocols to construct complexion TTT diagrams are not yet developed. Thus, the first challenge is to explore and exploit modern techniques to study the nucleation and growth of complexion transitions. We note that techniques that measure bulk properties may not necessarily be useful in this context. Furthermore, the materials systems that will be most amenable to the development of complexion TTT diagrams are those that, during coarsening, exhibit readily measurable changes in grain-boundary structure, chemistry, and properties. Ultimately, the newly developed experimental methods for generating reliable complexion TTT diagrams must answer several open questions:

1. What are the nucleation sites for complexion transitions (e.g. surfaces, grain boundaries, or triple lines)?

Recommendation: In order to answer this question it is imperative to develop methods capable of revealing the nucleation sites (grain boundaries, triple lines, second phase particles, pores, etc.) in real microstructures. Small volumes of material that contain individual grain boundaries or grain boundaries connected to a single triple line could be separated from a bulk polycrystal with the aid of focused-ion beam (FIB) milling techniques. These small volumes could be studied to determine whether the triple lines or grain boundaries are preferential nucleation sites. Transitions at these individual boundaries could also be studied with the aid of EBSD, capturing the important dependence of complexion transitions upon grain-boundary character. 
2. What is the relative degree of influence of thermodynamic parameters (e.g. temperature and pressure) and grain-boundary character (i.e., the five macroscopic degrees of freedom) in nucleating a complexion transition?

Recommendation: To expand the utility of the experiment suggested above, the chemistry and crystallographic texture of the bulk sample can be changed to study the influence of additives and the relative amount of specific grain boundary types. Additional solute can be added to induce the precipitation of secondary particles that act as atomic reservoirs or sinks, and the most common grain boundaries (e.g. $\Sigma 3$ ) can be isolated and studied as a function of these parameters.

3. Does the starting microstructure influence the onset of a complexion transition (i.e. does average grain size dictate the ratio between grain boundaries and triple lines)?

Recommendation: If triple lines are the predominant nucleation sites for complexion transitions, one can imagine that the grain size of the starting microstructure will influence the kinetics of complexion transitions, because microstructures with different grain sizes will have different ratios of grain boundary area to triple line density. For example, an experiment could be developed in which the starting grain size is tailored; the smaller the grain size, the more relevant will be the triple lines in catalyzing nucleation. If the triple lines are the preferred nucleation sites for complexion transitions, then the complexion TTT curves should shift to the left with decreasing grain size since there is a higher concentration of nucleation sites, i.e. complexion transitions should propagate more rapidly.

4. Besides the observation of abnormal grain growth, what other methods can be used to study the kinetics of grain-boundary complexion transitions in real polycrystals, and how can this information be used to generate complexion TTT diagrams?

Recommendation: The overarching challenge to advance our understanding of complexion transformation kinetics is to gain the ability to detect complexion transitions, via abrupt grain-boundary property changes, in real time at elevated temperatures. Abnormal grain growth is most often used as a proxy of a complexion transition, but it is a delayed indicator. The very nature of abnormal grain growth requires time for a grain to grow abnormally larger than the surrounding grains, thus blurring the true start of the complexion transformation. A few potential methods to 
detect a complexion transition include: grain-boundary impedance measurements to measure alterations in resistivity [25], Raman spectroscopy to measure differences in vibrational frequency of a grain boundary [26], and calorimetry experiments to detect heat given off during a transformation since complexion transitions reduce the free energy of the system (i.e. complexion transitions are exothermic) [27].

\section{What is the best method to compare grain-boundary structures before and after a complexion transition?}

Recommendation: Perhaps the most useful method to study the structural differences associated with grain-boundary complexions is transmission electron microscopy (TEM). Significant progress has been made with regard to studying grain-boundary structure and chemistry with the development of aberration-corrected electron microscopy. Aberration correction has been accessible for the past 15 years and, as a result, internal interfaces are now observed on the atomic scale [28]. Furthermore, with recent improvements in in-situ capabilities [29] it is now possible to observe grainboundary phenomena (e.g. grain growth) in real time at elevated temperatures. Therefore, in-situ electron microscopy should be utilized to observe complexion transitions as they occur in real time. Assuming complexion transitions are reversible, it may even be possible to determine quantitatively the nucleation rates if the TEM specimen is cycled several times above and below the transition temperature. It may also be possible to deduce differences in structure between grain boundary segments near a triple line and segments farther away. If triple points are nucleation sites for complexion transitions, in-situ experiments may be the most effective methods to identify the sites and characterize the time dependence of the nucleation process. Amongst electron microscopy methods, there are several potentially advantageous techniques that have been recently reviewed by Robertson et al. [30]. They summarized a state-of-the-art integrated materials characterization toolbox that includes electron microcopy, atom probe tomography, and diffraction-based analysis techniques to study materials in both the spatial and temporal domains. We suggest that this toolbox and similar techniques be used to study complexion transitions in real time. 


\section{In principle, each type of grain boundary should have its own curve on a complexion}

TTT diagram, but in practice some simplification is necessary. What are the best ways to represent the wide variety of grain-boundary complexion transition behavior on a single complexion TTT diagram?

Recommendation: In real polycrystals, each grain boundary behaves at least somewhat differently from the others, and no two grain boundaries are exactly alike. Complexion transitions will occur at different times and temperatures on different grain boundaries depending upon their grain-boundary character (i.e., the five macroscopic degrees of freedom). However, plotting a set of transition curves on a complexion TTT diagram for each type of grain boundary would be impractical and unnecessarily complex. In some cases, all of the grain boundaries behave similarly enough that a single set of TTT curves can represent the overall behavior, as shown in Figure 7. In other cases, such as in highly anistropic systems that easily adopt bimodal microstructures, subsets of grain boundaries with similar behavior can be lumped together to produce sets of curves that represent each general type of grain-boundary behavior. This type of complexion TTT diagram is shown schematically in Figure 2.

\section{What are the most efficient means to study the kinetics of complexion transitions and} catalogue a large number of transitions for various materials systems?

Recommendation: Experimental approaches like those presented above are extremely valuable, but limited by time and sample volume constraints. On the other hand, computer simulations have the capability to survey more materials systems and are constantly proving to be equal counterparts to investigate complexion transitions. With the advent of high-performance computing, it is now possible to simulate larger microstructures that more accurately represent real materials. Therefore, computational efforts can be used to determine complexion nucleation rates, build Avrami curves, and construct associated TTT diagrams. For instance, large-scale molecular dynamics (MD) simulations of a generated microstructure may be employed to study complexion nucleation via the manipulation of temperature and composition. The results of these simulations can then inform mesoscale simulations that model the evolution of a complexion transition at longer time scales [31]. In addition, MD simulations complement electron microscopy experiments as both can be used to study the atomic 
structure of complexions, and both can therefore be used to validate each other. Lastly, Potts model simulations can also be employed to examine possible complexion propagation (growth) scenarios and the resulting microstructures.

\section{SUMMARY}

Until recently, complexion transition kinetics have been largely overlooked by the materials community, despite their strong influence on the behavior of bulk polycrystalline materials. We have presented an experimental study in polycrystalline Eu-doped spinel that demonstrates that grain-boundary complexion transition kinetics can be represented on a complexion TTT diagram. In many ways, complexion TTT diagrams are analogous to bulk phase TTT diagrams, and they promise to be similarly useful tools for optimizing the performance of polycrystalline materials via carefully chosen heat treatment protocols. We have pointed out several challenges that are typically encountered while generating experimental complexion TTT diagrams and made recommendations for overcoming these challenges. In particular, theoretical modeling and computer simulation will be helpful in the development of experimental complexion TTT diagrams. It is anticipated that complexion TTT diagrams will be remarkably useful tools for tailoring the microstructure and, hence, the performance of bulk polycrystalline metals and ceramics. The field of complexion TTT diagrams is just beginning, and the compilation of many more complexion TTT diagrams for various materials systems will be required before the full potential of these diagrams can be realized.

\section{Acknowledgments}

The authors gratefully acknowledge support from ONR-MURI program (grant no. N0001411-0678). Partial support is acknowledged from the NSF under CMMI-1436585.

\section{REFERENCES}

[1] E.S. Davenport, E.C. Bain, Transformation of austenite at constant subcritical temperatures, Trans. AIME. 90 (1930) 117-144. 
[2] M.P. Harmer, The phase behavior of interfaces, Science. 332 (2011) 182-183. doi:10.1126/science.1204204.

[3] P.R. Cantwell, M. Tang, S.J. Dillon, J. Luo, G.S. Rohrer, M.P. Harmer, Grain boundary complexions, Acta Mater. 62 (2014) 1-48. doi:10.1016/j.actamat.2013.07.037.

[4] W.D. Kaplan, D. Chatain, P. Wynblatt, W.C. Carter, A review of wetting versus adsorption, complexions, and related phenomena: the rosetta stone of wetting, J Mater Sci. 48 (2013) 5681-5717. doi:10.1007/s10853-013-7462-y.

[5] P.R. Cantwell, S. Ma, S. Bojarski, G. Rohrer, M.P. Harmer, Expanding time-temperaturetransformation (TTT) diagrams to interfaces: A new approach for grain boundary engineering, Acta Materialia. 106 (2016) 78-86. doi:10.1016/j.actamat.2016.01.010.

[6] S.J. Dillon, M. Tang, W.C. Carter, M.P. Harmer, Complexion: A new concept for kinetic engineering in materials science, Acta Mater. 55 (2007) 6208-6218. doi:10.1016/j.actamat.2007.07.029.

[7] S.J. Dillon, M.P. Harmer, Demystifying the role of sintering additives with "complexion," Journal of the European Ceramic Society. 28 (2008) 1485-1493. doi:10.1016/j.jeurceramsoc.2007.12.018.

[8] J.B. Hannon, H. Hibino, N.C. Bartelt, B.S. Swartzentruber, T. Ogino, G.L. Kellogg, Dynamics of the silicon (111) surface phase transition, Nature. 405 (2000) 552-554. doi:10.1038/35014569.

[9] S.A. Bojarski, M.P. Harmer, G.S. Rohrer, Influence of grain boundary energy on the nucleation of complexion transitions, Scripta Mater. 88 (2014) 1-4. doi:10.1016/j.scriptamat.2014.06.016.

[10] T. Watanabe, An approach to grain boundary design of strong and ductile polycrystals, Res. Mech. 11 (1984) 47-84.

[11] M.P. Harmer, Interfacial Kinetic Engineering: How Far Have We Come Since Kingery's Inaugural Sosman Address?, Journal of the American Ceramic Society. 93 (2010) 301-317. doi:10.1111/j.1551-2916.2009.03545.x.

[12] S.J. Dillon, H. Miller, M.P. Harmer, G.S. Rohrer, Grain boundary plane distributions in aluminas evolving by normal and abnormal grain growth and displaying different complexions, IJMR. 101 (2010) 50-56. doi:10.3139/146.110253.

[13] S.A. Bojarski, S. Ma, W. Lenthe, M.P. Harmer, G.S. Rohrer, Changes in the Grain Boundary Character and Energy Distributions Resulting from a Complexion Transition in CaDoped Yttria, Metall and Mat Trans A. 43 (2012) 3532-3538. doi:10.1007/s11661-012-1172y.

[14] D.M. Saylor, B.S. El-Dasher, B.L. Adams, G.S. Rohrer, Measuring the five-parameter grain-boundary distribution from observations of planar sections, Metall and Mat Trans $A$. 35 (2004) 1981-1989. doi:10.1007/s11661-004-0147-z.

[15] M. Avrami, Kinetics of Phase Change. I General Theory, The Journal of Chemical Physics. 7 (1939) 1103. doi:10.1063/1.1750380.

[16] K. Sekimoto, Evolution of the domain structure during the nucleation-and-growth process with non-conserved order parameter, Physica A: Statistical Mechanics and Its Applications. 135 (1986) 328-346. doi:10.1016/0378-4371(86)90146-9.

[17] J.M. Rickman, W.S. Tong, K. Barmak, Impact of heterogeneous boundary nucleation on transformation kinetics and microstructure, Acta Materialia. 45 (1997) 1153-1166. 
[18] C.P. Khattak, F. Schmid, Growth of the world's largest sapphire crystals, Journal of Crystal Growth. 225 (2001) 572-579. doi:10.1016/S0022-0248(01)00955-1.

[19] M.S. Akselrod, F.J. Bruni, Modern trends in crystal growth and new applications of sapphire, Journal of Crystal Growth. 360 (2012) 134-145. doi:10.1016/j.jcrysgro.2011.12.038.

[20] B. Raeisinia, C.W. Sinclair, W.J. Poole, C.N. Tomé, On the impact of grain size distribution on the plastic behaviour of polycrystalline metals, Modelling Simul. Mater. Sci. Eng. 16 (2008) 025001. doi:10.1088/0965-0393/16/2/025001.

[21] M.A. Meyers, A. Mishra, D.J. Benson, Mechanical properties of nanocrystalline materials, Progress in Materials Science. 51 (2006) 427-556. doi:10.1016/j.pmatsci.2005.08.003.

[22] I. W. Chen, X. H. Wang, Sintering dense nanocrystalline ceramics without final-stage grain growth, Nature. 404 (2000) 168-171. doi:10.1038/35004548.

[23] S.-J.L. Kang, J.-H. Park, S.-Y. Ko, H.-Y. Lee, Solid-State Conversion of Single Crystals: The Principle and the State-of-the-Art, J. Am. Ceram. Soc. 98 (2015) 347-360. doi:10.1111/jace.13420.

[24] J. Czochralski, Process of improving alloys and metals, US1560335 A, 1925. http://www.google.com/patents/US1560335 (accessed December 22, 2015).

[25] M. Gerstl, E. Navickas, G. Friedbacher, F. Kubel, M. Ahrens, J. Fleig, The separation of grain and grain boundary impedance in thin yttria stabilized zirconia (YSZ) layers, Solid State Ionics. 185 (2011) 32-41. doi:10.1016/j.ssi.2011.01.008.

[26] S. Veprek, F.-A. Sarott, Z. Iqbal, Effect of grain boundaries on the Raman spectra, optical absorption, and elastic light scattering in nanometer-sized crystalline silicon, Phys. Rev. B. 36 (1987) 3344-3350. doi:10.1103/PhysRevB.36.3344.

[27] G.C.C. Costa, S.V. Ushakov, R.H.R. Castro, A. Navrotsky, R. Muccillo, Calorimetric Measurement of Surface and Interface Enthalpies of Yttria-Stabilized Zirconia (YSZ), Chemistry of Materials. 22 (2010) 2937-2945. doi:10.1021/cm100255u.

[28] D.J. Smith, Development of Aberration-Corrected Electron Microscopy, Microscopy and Microanalysis. 14 (2008) 2-15. doi:10.1017/S1431927608080124.

[29] H. Zheng, Y.S. Meng, Y. Zhu, Frontiers of in situ electron microscopy, MRS Bulletin. 40 (2015) 12-18. doi:10.1557/mrs.2014.305.

[30] I.M. Robertson, C.A. Schuh, J.S. Vetrano, N.D. Browning, D.P. Field, D.J. Jensen, M.K. Miller, I. Baker, D.C. Dunand, R. Dunin-Borkowski, B. Kabius, T. Kelly, S. Lozano-Perez, A. Misra, G.S. Rohrer, A.D. Rollett, M.L. Taheri, G.B. Thompson, M. Uchic, X.-L. Wang, G. Was, Towards an integrated materials characterization toolbox, Journal of Materials Research. 26 (2011) 1341-1383. doi:10.1557/jmr.2011.41.

[31] W.E. Frazier, G.S. Rohrer, A.D. Rollett, Abnormal grain growth in the Potts model incorporating grain boundary complexion transitions that increase the mobility of individual boundaries, Acta Materialia. 96 (2015) 390-398. doi:10.1016/j.actamat.2015.06.033. 